\title{
A Case of Recurrent Stroke in Left MCA Stenosis
}

\author{
Bhuiyan A A 1 , Shahriar S M H², Nahid F3, Alam M J4
}

\begin{abstract}
We report the clinical, neuro-radiological findings of a young man who was admitted to Apollo Hospitals Dhaka, Bangladesh because of abrupt onset of aphasia and right sided mild hemiparesis. He had two strokes six days apart one and half years ago. His past medical history was significant for HTN, smoking. His MRI of the Brain showed acute infarct in left middle cerebral artery (MCA) region congruent with his symptoms and signs. MRA done during his first stroke showed left MCA long segment luminal narrowing. DSA done during initial stroke showed complete occlusion of superior division of left MCA with collateral flow from left anterior cerebral artery (ACA) and posterior cerebral artery (PCA) to left MCA. Other extensive work up for stroke etiology was inconclusive. His stroke recurrence points towards left MCA stenosis (which is reversible) with good collateral circulation which helped the patient get significant neurological recovery.
\end{abstract}

In young patients with recurrent strokes with or without conventional risk factors, vascular anomalies (intra-cranial and extra-cranial) should be investigated to find out the other rare causes for strokes. As TEE was not done here, it conclusively can not be ascertained that some cardio-embolic source of stroke was not present.

\section{Introduction}

The occurrence of middle cerebral artery (MCA) stenosis as a risk factor of stroke is more frequent in eastern than western countries. Atherosclerotic intracranial artery stenosis occurs most frequently in the MCA region. The MCA stenosis causes brain infarction and is also a risk factor of recurrent stroke. ${ }^{1}$ Stenosis of higher than $70 \%$ grade represented as $>70 \%$ stenosis, results in a yearly recurrence rate of $23 \% .^{2}$ Intracranial large artery atherosclerosis causes approximately $10 \%$ of all ischaemic strokes in the United States. ${ }^{3}$ In studies of MCA occlusion on the other hand the sexes have been roughly equal.

Even in patients with conventional risk factor for stroke, vascular studies (both intracranial and extra cranial) should be done to look for vascular anomalies. The later, can rather be most important contributing factor for stroke. If stroke occur due to vascular anomalies, the strokes can be recurrent, occur in the same vascular distribution and has significant neurological recovery.

The case presented here is regarding a young Bangladeshi male with two conventional risk factors (HTN and smoking) associated with left MCA stenosis. Patient had recurrent strokes (three times) in the same vascular distribution and had significant neurological recovery.

\section{Case Presentation}

$\mathrm{MH}$ is 42 years old Bangladeshi male, right handed was admitted in Apollo Hospital Dhaka 
on 29/04/13 with two day history of dysphasia and mild right sided weakness involving the upper extremity. He had some difficulty with understanding and expression of language with associated anomic and paraphasic errors.

He had two strokes six days apart one and half years ago when he had inability to speak (expressive aphasia) and right sided weakness (1/5 in upper and lower extremity). He recovered significantly slowly to motor strength $4 / 5$.

He had history of HTN and smoking. He had no history of DM, cardiac disease, rheumatological disease, haematological disease or history of recent travel.

He had no h/o alcohol abuse or illicit drug use. He has h/o heavy smoking (1 pack year). His F/H was unremarkable. He had no known drug allergy. He worked as a manager in a garments buying house. He was taking Atenolol 50 mg QD and Aspirin 75 mg QD.

On examination his BP was - 140/90, pulse-72 (regular), afebrile, RR-14/minute. His weight was $62 \mathrm{~kg}$. Neurological examination: Mental status exam showed-he was alert, awake. He scored 29/30 on MSSE (recall was 2/3). On Montreal cognitive assessment (MOCA) he scored 18/30 (missed on abstraction, delayed recall and trail making test). Cranial nerve exam was only significant for right UMN type VII nerve palsy. Motor exam showed 5/5 in all upper limbs without any atrophy.

Muscle tone was normal and there was no atrophy. No cerebellar abnormality was noted. Reflexes were 2, symmetric on both sides and both toes showed planter flexion.

Sensory exam showed no abnormality to all modalities. Gait was steady. He had no carotid bruits. Systemic exam was normal without any skin stigmata. MRI of the brain showed acute infarct in left capsulo-ganglionic and parietal region (in congruent with patients findings), (Fig I and II).

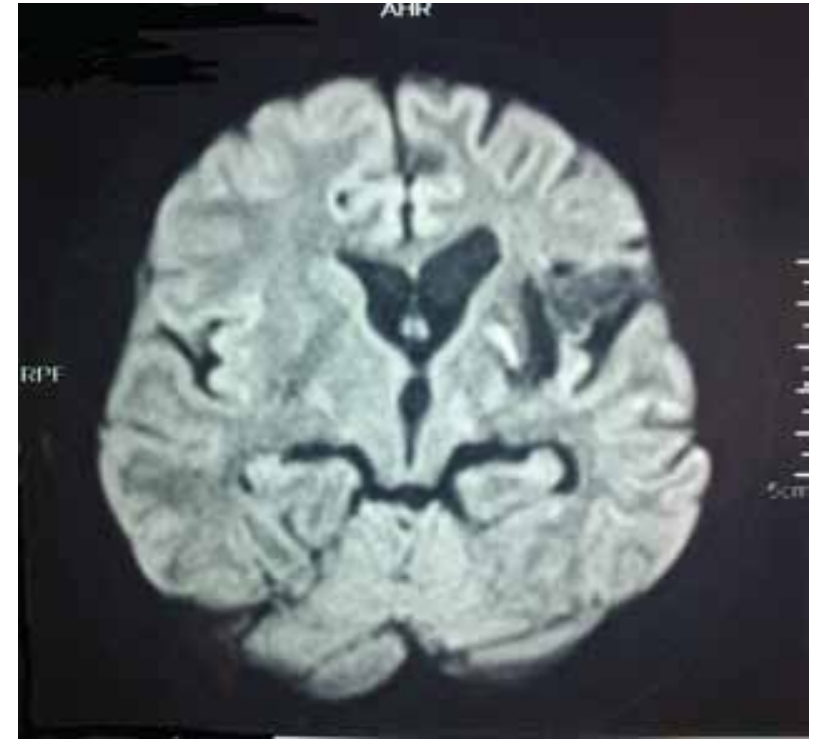

Fig: I

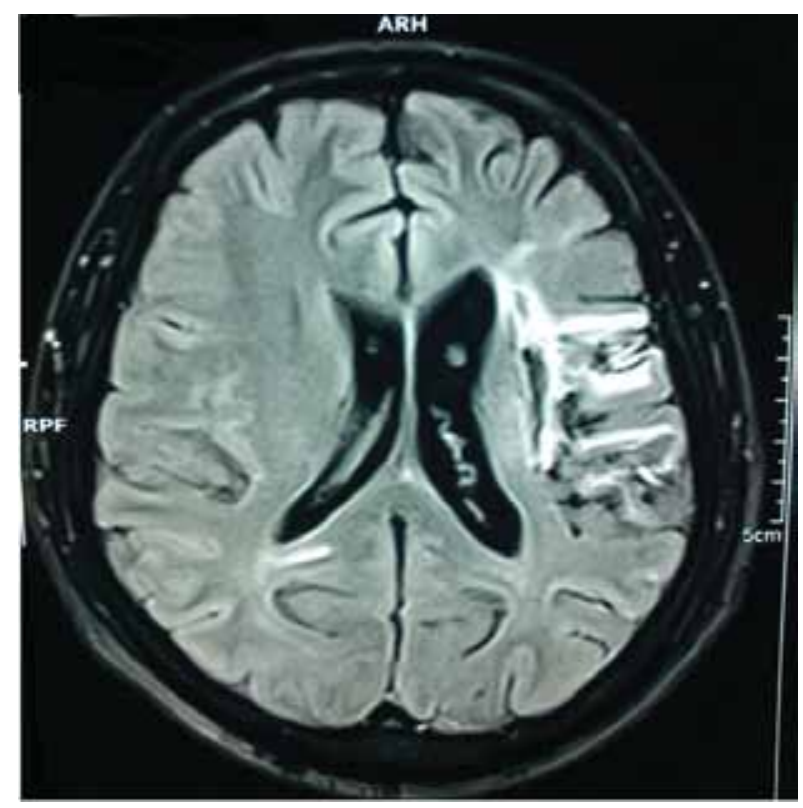

Fig: II

Carotid Doppler shows 35-50\% diameter reduction of left distal common carotid artery (at the bulb) by short segment fibro-fatty plaque,haemodynamically non-significant fibro-fatty plaque in right distal common carotid artery causing 15-25 \% luminal diameter reduction. 


\section{CASE REPORT}

MRA showed marked left MCA luminal narrowing for a long segment extending from its

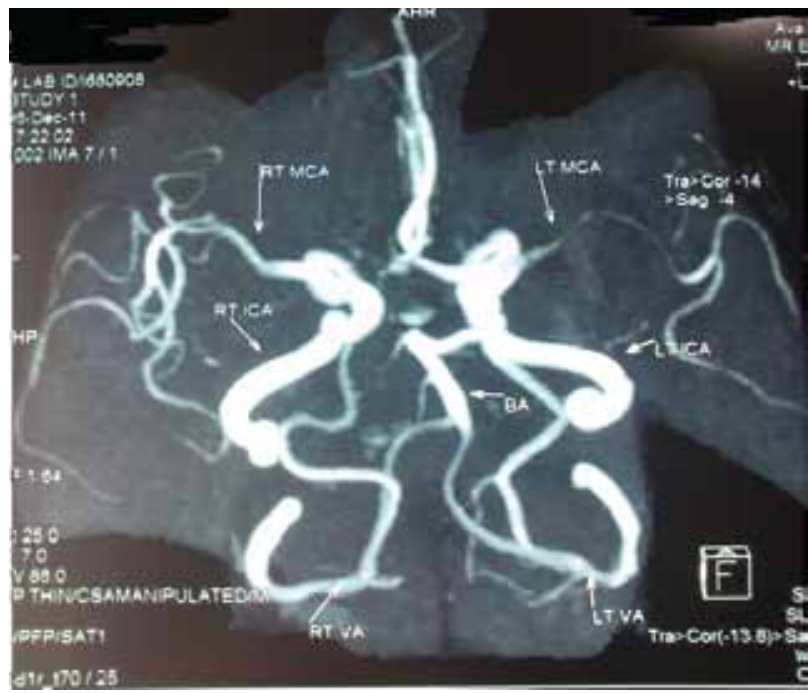

Fig: III

His cerebral DSA (Fig. IV) done in an out side hospital abroad showed complete occlusion of superior division of left MCA with collateral flow from left ACA and PCA to left MCA territory. Echocardiogram was unremarkable.

His serum Electrolytes, CBC and ESR, S. Creatinine, LFT including Serum ANA, Protein C, Protein S, Anti-thrombin III, Anti phospholipid antibody was unremarkable. HIV I and II, VDRL, CRP, Hepatitis B surface Antigen, HCV IgG Ab was negative. Trans esophageal echocardiogram and Trans-cranial Doppler (not available) was not done.

Anti-platelet agent (Aspirin 75 mg / day) and anti-lipid drug (HMG Co-A reductase inhibitor) was started after first stroke. After his last stroke he was prescribed warfarin with adjustment of his INR around 2. He was given physio-therapy and speech therapy.

\section{Discussions}

Although several studies have examined the risk factors and rate of recurrence in each subtype of origin with attenuation of peripheral arteries, (Fig III).

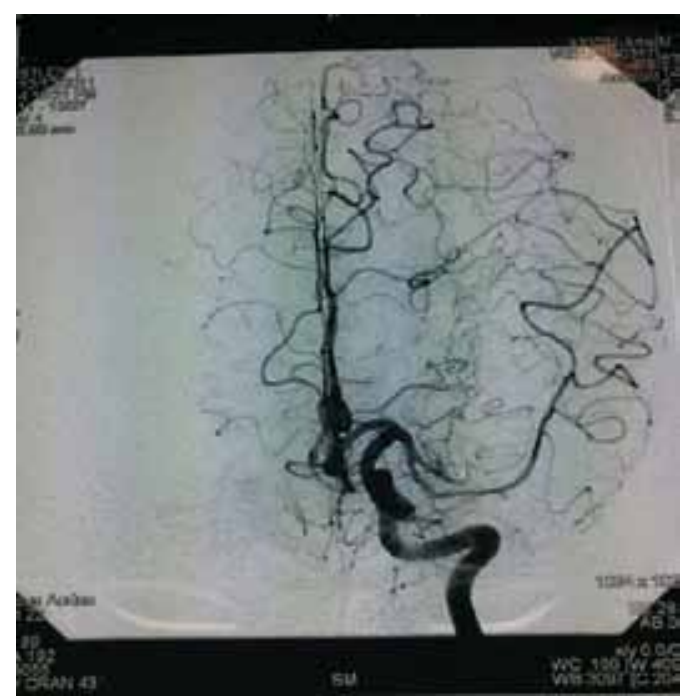

Fig: IV

ischaemic stroke, the mechanism of recurrent stroke in Asians are unclear and may differ from those in westerners because there are racial differences in the risk factor profiles and distribution of occlusive disease. ${ }^{4}$ It seems that intra-cranial internal carotid artery stenosis is a better marker of severe generalized vascular disease than is MCA stenosis.

The mechanisms of the index and recurrent stroke using the clinical syndrome, diffusion-weighted imaging, and vascular strokes, divided patients into 5 groups: large artery atherosclerosis, cardio-embolism, small artery disease, parent-artery disease occurring near the deep perforators and no determined cause. The patients with large artery atherosclerosis were sub-divided into 2 groups: intra-cranial and extra-cranial.

Extra-cranial large artery atherosclerosis (LAA) is a form of systemic atherosclerosis and that plaque stability rather than the degree of stenosis is more important in this is ischaemic mechanism where as intracranial LAA is a form 
of local (intracranial) disease or arteriosclerosis. In patients with stroke and middle cerebral artery lesions, progression of stenosis was associated with an increased risk of transient ischaemic attack or ischaemic stroke but not acute coronary syndrome. ${ }^{5}$ Several previous studies showed that intracranial atherosclerosis is a highly dynamic process, reporting both progression or regression by using neuroradiological ${ }^{6}$ and neuro-sonological technique. ${ }^{7}$ The conclusion of all studies is that the progression of a symptomatic MCA stenosis is a strong predictor of stroke recurrence. ${ }^{8}$

In ethnic groups with a high prevalence of intracranial stenosis, such as Asians, the progression of intracranial LAA from non significant $(<50 \%)$ to significant $(>50 \%)$ may account for the recurrent stroke in patients with the small artery disease (SAD) and no apparent cause groups. 9

Intracranial stenosis accounted for the recurrent stroke in some patients in the SAD groups; this finding is supported by reports that a significant fraction of patients with small deep infarcts had large intracranial arterial lesions occluding the orifices of the perforators. ${ }^{10}$ In the case of recurrent brain infarction by MCA stenosis, the possibility is that hypo-perfusion as well as distal embolism plays an important part.

The patient history described shows that he had the typical atherosclerotic features of stroke like HTN and smoking. He had no h/o illicit-drug use or features of hypercoagulable state (normal PT, INR, aPTT), hyper viscosity syndrome (Negative S. Protein C, Protein S, Anti-thrombin III deficiency) or rheumatologic causes (Negative ANA). His strokes occurred at relatively young age. His recurrent stroke symptoms happened in the same vascular distribution. He had significant recovery after each strokes without major residual neurological deficit. His cerebral DSA showing complete occlusion of superior division of left MCA with collateral flow from left ACA and PCA to left MCA territory.

Now a day's medical treatment for patients with $>50 \%$ stenosis intracranial arteries may be followed by a recurrence rate of $20-25 \%$ per year. Prognosis for recovery from the initial stroke is, however, better in MCA stenosis than that formed in stenosis of MCA occlusion. This compares with good recovery of $71 \%$ of series of Cornston et $\mathrm{al}^{11}$ and $88 \%$ of the series of Hinton el-al. ${ }^{12}$

He was given medical management for his condition. This case shows that the presence of both typical risk factors such as HTN and smoking along with non-traditional risk factor i.e left MCA stenosis was present, although the later was the predominant risk factor for the recurrent strokes. He did not have Trans oesophageal Echocardiogram to look further for cardio-embolic source.

\section{Conclusion}

In young patient with stroke traditional risk factors like HTN, smoking along with vascular anomalies (extra-cranial and intra-cranial) can be present. So investigation should also include vascular work-up involving intra cranial and extra cranial blood vessels. In young south asian patients with stroke, vascular anomalies is a very important risk factor. It is true especially in case of recurrent strokes and if a stroke happens in the same vascular distribution with significant neurological recovery. TEE should always be done to look for additional cardio-embolic source. Further studies should be done to determine the contribution of different risk factors for strokes, their combined patho-physiology and prognosis. 


\section{CASE REPORT}

\section{References}

1. Chimowitz MI, Lynn MJ, Howlett-Smith $\mathrm{H}$. Comparison of warferin and aspirin for symptomatic intracranial arterial stenosis. Neurology. 2008;70:1518-1524.

2. Zaidat OO, Klucznik R, Alexander MJ. The NIH registry on use of the wingspan stent for symptomatic 70-99\% intracranial arterial stenosis. Neurology. 2008;70:1518-1524.

3. Sacco RL, Kargman DE Gu, QZamanillo MC. Race ethnicity and determinants of intracranial atherosclerotic cerebral infraction: the northern manhatten stroke study. Stroke. 1995;2614-20.

4. Bogousslavsky J, Barnett HJM, Fox AJ, Hachinski VC, Taylor W. Atherosclerotic disease of the middle cerebral artery. Stroke. 1986;17:1112-1120.

5. Wong KS, Li H, Lam W W, Chan YL, Kay R. Progression of middle cerebral artery occlusive disease and its relationship with further vascular events after Stroke. Stroke. 2002;3;3532-3536.

6. Akins PT, Pilgram TK, Cross DT, Moran CJ: Natural history of stenosis from intracranial atherosclerosis by serial sngiography. Stroke 1998;29:433-438.

7. Arenillas JF, Montaner J, Abilleira S, González-Sánchez MA, Álvarez-Sabín J.
Progression and clinical recurrence of symptomatic middle cerebral artery stenosis: a long-term follow-up transcranial doppler ultrasound study. Stroke. 2001;32:2898-2904.

8. Malferrari G, Bertolino C, Casoni F, Zini A, Sarra VM, Sanguigni $S$ et al. For the Eligible group and SINV group: The Eligible Study: Ultrasound assessment in acute ischemic stroke within 3 Hours. Cerebrovasc Dis. 2007;24:469-76.

9. Shin DH, Lee PH, Bang OY. Mechanism of recurrence in subtypes of ischemic stroke: a hospital-based follow-up study. Arch Neurol. 2005;62(8):1232-1337.

10. Bang OY, Heo JH, Kim JY, Park JH, Huh K. Middle cerebral artery stenosis is a major clinical determinant in striatocapsular small, deep infarction. Arch Neurol. 2002;59:259-263.

11. Corston RN, Kendall BE, Marshall J: Prognosis in middle cerebral artery stenosis. Stroke 1984:15:237-241.

12. Hinton RL, Mohr JP, Ackerman RH, Adair CB, Fisher CM. Symptomatic middle cerebral artery stenosis Ann Neurology. 1979;5:152-157. 